\title{
Modelo ABC para o manejo da úlcera venosa de perna
}

\author{
$A B C$ model for leg ulcer management
}

Modelo ABC para el manejo de la úlcera venosa de pierna

Eline Lima Borges', Camyle de Melo dos Santos², Mariana Raquel Soares 2,3

\begin{abstract}
RESUMO
Os profissionais de saúde necessitam de aportes teórico e prático para que possam fazer, de forma efetiva, recomendações de tratamentos específicos aos pacientes com úlcera venosa, bem como desmitificar a utilização da terapia de compressão. O estudo objetivou apresentar a utilização do Modelo ABC no gerenciamento de úlcera venosa de perna. Trata-se de uma atualização concernente à avaliação sistematizada e indicação do tratamento amparadas no Modelo ABC no manejo de úlcera, que compreende: (A) avaliação e diagnóstico; (B) boas práticas no manejo da ferida e da pele; e (C) compressão na otimização do tratamento da lesão e prevenção de recidivas. As etapas A, B e C podem ser sintetizadas com a apresentação de quatro, cinco e sete recomendações, respectivamente. A adoção do Modelo permite que o profissional siga um percurso sistematizado para o tratamento do paciente com úlcera venosa. Espera-se que o modelo proposto com abordagem simplificada auxilie os clínicos profissionais a entenderem claramente por que, quando e como a terapia de compressão deve ser utilizada.
\end{abstract}

DESCRITORES: Úlcera varicosa. Bandagens compressivas. Avaliação em saúde. Estomaterapia.

\begin{abstract}
Health professionals need theoretical and practical contributions so that they can effectively recommend specific treatments to patients with venous ulcers and demystify the use of the compression therapy. This study focused on using the ABC Model for leg ulcer management. It is an update concerning the systematic evaluation and treatment indication supported by the ABC Model for ulcer management that includes: (A) evaluation and diagnosis; (B) best practices on wound and skin management; and (C) compression therapy for active treatment and prevention of recurrence. Steps A, B and C may be summarized by presenting four, five and seven recommendations, respectively. The adoption of the Model allows the professional to follow a systematic path in the treatment of patients with venous ulcers. It is expected that the proposed Model with simplified approach helps clinicians to clearly understand why, when and how compression therapy should be used.
\end{abstract}

DESCRIPTORS: Varicose ulcer. Compression bandages. Health evaluation. Stomatherapy.

\section{RESUMEN}

Los profesionales de salud necesitan de aportes teórico y práctico para que puedan realizar, de forma efectiva, la recomendación de tratamientos específicos a los pacientes con úlcera venosa, así como la desmitificación de la utilización de la terapia de compresión. Este estudio tuvo por objeto la utilización del Modelo ABC en el gerenciamiento de la úlcera venosa de pierna. Se trata de una actualización de la evaluación sistematizada e indicación del tratamiento, amparadas en el Modelo ABC en el manejo de úlcera, que comprende: (A) evaluación y diagnóstico; (B) buenas

${ }^{1}$ Departamento de Enfermagem Básica da Escola de Enfermagem da Universidade Federal de Minas Gerais (UFMG) - Belo Horizonte (MG), Brasil. Endereço para correspondência: Escola de Enfermagem da Universidade Federal de Minas Gerais - Avenida Professor Alfredo Balena, 190 -

Santa Efigênia - CEP: 30130-100 - Belo Horizonte (MG), Brasil - E-mail: eborges@ufmg.br

$23 \mathrm{M}$ do Brasil - Rio de Janeiro (RJ), Brasil.

$33 \mathrm{M}$ do Brasil - Belo Horizonte $(\mathrm{BH})$, Brasil.

Artigo recebido em: 27/04/2016 - Aceito para publicação em: 28/07/2016. 
prácticas en el manejo de la herida y de la piel; y (C) comprensión en la optimización del tratamiento de la lesión y prevención de recurrencias. Las etapas A, B y C pueden ser sintetizadas con la presentación de cuatro, cinco y siete recomendaciones, respectivamente. La adopción del Modelo permite que el profesional continúe una ruta sistematizada para el tratamiento del paciente con úlcera venosa. Se espera que el modelo propuesto con enfoque simplificado ayude a los profesionales clínicos a entender claramente por qué, cuándo y cómo la terapia de la compresión debe ser utilizada.

DESCRIPTORES: Úlcera varicosa. Vendajes de compresión. Evaluación en salud. Estomaterapia.

\section{INTRODUÇÃO}

A perna pode ser acometida por úlceras de diversos tipos e etiologias, sendo mais frequentes as decorrentes de doença venosa, doença arterial, neuropatia, e outras causas menos comuns como devido a distúrbios metabólicos, hematológicos e doenças infecciosas ${ }^{1}$. Tem sido relatado que $70,0 \%$ das úlceras de perna estão relacionadas à insuficiência venosa, 10,0\% à doença arterial e 15,0\% das apresentações são de etiologia mista. Os 5,0\% restantes têm causas fisiopatológicas menos comuns, e este último grupo compreende desafios consideráveis no diagnóstico, na avaliação e na gestão ${ }^{2}$.

Cerca de 60,0 a 80,0\% das úlceras de perna contêm um componente venoso, e sua prevalência aumenta com a idade. Ressalta-se que no Reino Unido a úlcera varicosa tem prevalência estimada entre 0,1 e $0,3 \%^{3}$, e nos Estados Unidos a prevalência global é de 1,0\% aproximadamente ${ }^{1}$. As úlceras varicosas são mais comuns em mulheres e em pessoas com 65 anos ou mais, e os principais fatores de risco são trombose venosa profunda, flebite, lesão anterior na perna e obesidade 4 .

Apesar da recomendação existente sobre a utilização da terapia de compressão para o aumento da taxa de cicatrização da úlcera, muitos pacientes que apresentam esse tipo de lesão não recebem essa modalidade de terapia. No Reino Unido, apenas 20,0\% dos pacientes da atenção primária com úlcera venosa receberam a terapia de compressão. Na França, apenas 10,8\% dos médicos de clínica geral seguiram orientações para a gestão de úlcera venosa. Entretanto, nos centros especializados desse mesmo país, a terapia de compressão pode ser utilizada em até $88,0 \%$ dos pacientes com úlcera venosa. $\mathrm{Na}$ Alemanha, uma companhia de seguros informou que 32,0 a 53,0\% dos pacientes com esse tipo de úlcera receberam terapia de compressão ${ }^{5}$.

Existem inúmeras razões para que a terapia de compressão não seja utilizada pelos profissionais no tratamento de úlcera varicosa, tais como: falta de conhecimento ou confiança dos médicos e enfermeiros; falta de referência e contra referência por causa da variedade de especialidades nas quais possam estar envolvidas; indisponibilidade de produtos; desconhecimento dos tipos de bandagens e de meias de compressão disponíveis no mercado; ausência de serviços especializados; e falta de adesão dos pacientes que utilizam terapia de compressão ${ }^{5}$.

Os profissionais de saúde necessitam de aportes teórico e prático para que possam fazer, de forma efetiva, recomendações de tratamentos específicos aos pacientes com úlcera venosa. São importantes a capacitação permanente dos profissionais que cuidam de pacientes com úlceras de perna e a garantia de acessibilidade aos recursos materiais necessários, visando reduzir a lacuna existente entre a prática assistencial e as evidências científicas, bem como a desmitificação da utilização da terapia compressiva.

\section{OBJETIVO}

Apresentar a utilização do Modelo ABC no gerenciamento da úlcera venosa de perna.

\section{MÉTODOS}

Trata-se de um estudo de atualização concernente à avaliação, às boas práticas e à compressão amparada no Modelo $\mathrm{ABC}$ no manejo de úlcera de perna.

O Modelo ABC foi elaborado por um grupo internacional de especialistas em úlceras de perna e doença venosa, durante reunião ocorrida em dezembro de 2014, na cidade de Londres. O grupo de trabalho foi constituído por dez profissionais da área de Enfermagem e Medicina de diversas 
especialidades e contou com dois revisores. Resultou no reconhecimento de que uma proporção muito elevada de todas as úlceras de perna tem doença venosa como um fator causal ou contributivo, ou seja, são úlceras venosas ou de etiologia mista; portanto, são apropriadas para a utilização de terapia de compressão.

As discussões dos especialistas estiveram centradas na identificação de como encorajar profissionais e pacientes a aumentarem a adoção da terapia de compressão no tratamento das úlceras, simplificando os princípios-chave envolvidos. As conclusões formaram a base do documento elaborado pelo grupo e são apresentadas como um $\mathrm{ABC}$ no manejo da úlcera venosa, com foco na fase de tratamento ativo. O documento produzido foi publicado em $2015 \mathrm{sob}$ o título "Simplifying venous leg ulcer management. Consensus recommendations".

O Modelo ABC (Figura 1) compreende:

A. avaliação e diagnóstico;

B. boas práticas no manejo da lesão e pele ao redor; e

C. compressão na otimização do tratamento das úlceras e prevenção de recidivas.

\section{RESULTADOS}

O Modelo ABC compreende as etapas "avaliação", "boas práticas na gestão da ferida/pele" e "compressão", que podem ser sintetizadas com a apresentação de quatro, cinco e sete recomendações, respectivamente (Figura 2). A adoção do

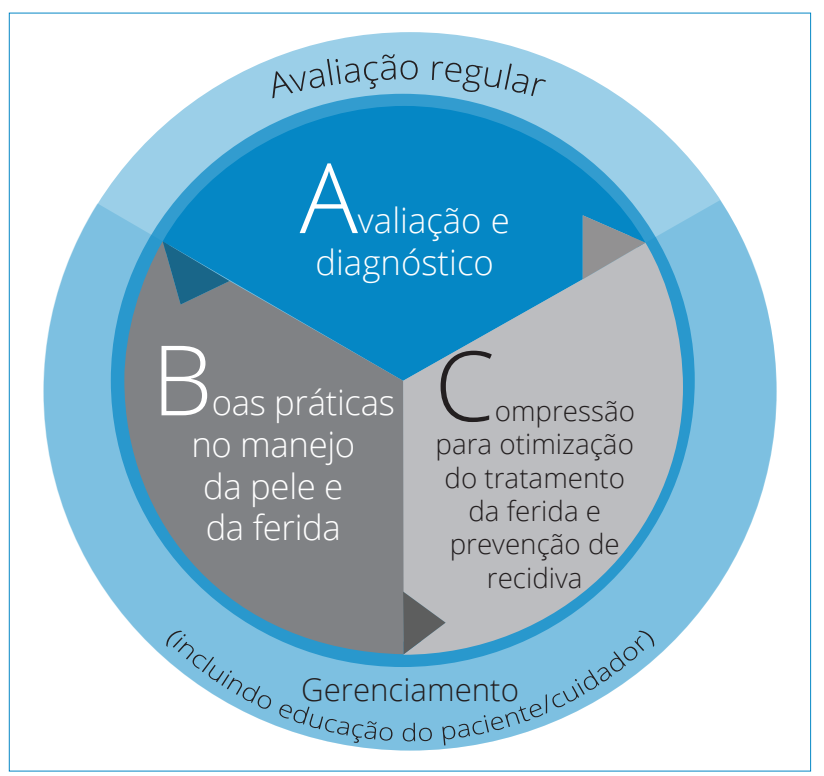

Figura 1. Modelo ABC. modelo permite que o profissional siga um percurso sistematizado para o tratamento do paciente com úlcera venosa (Figura 3).

\section{DISCUSSÃO}

Úlcera de perna é uma lesão crônica na pele, abaixo do nível do joelho, que persiste por mais de seis semanas e não mostra tendência para cicatrizar após 3 meses de tratamento adequado ou ainda não está totalmente curada em 12 meses $^{6}$. Pode ser definida como lesão com completa profundidade de espessura e uma tendência lenta de cura. Pode resultar na perda completa da epiderme e, muitas vezes, de porções da derme ou até mesmo do subcutâneo ${ }^{7}$. É uma alteração frequente entre os adultos; provoca dor física, estigma e preconceito, que reduzem o convívio social e provocam impactos negativos na qualidade de vida.

A úlcera de perna é considerada um problema de saúde pública, uma vez que a incidência tem crescido como resultado do envelhecimento da população e aumento dos fatores de risco para oclusão aterosclerótica, como o tabagismo, a obesidade e o diabetes ${ }^{7}$. Já é previsto que cerca de 1,0\% da população adulta terá uma úlcera de perna em algum momento de sua vida. Essa condição afeta 3,6\% das pessoas com mais de 65 anos $^{8}$. A prevalência está estimada entre 1,5 e 1,8 por 1.000 do total da população, e essa relação tende a aumentar com a idade para 3 por 1.000 na faixa etária de 61 a 70 anos e 20 por 1.000 em pessoas com idade superior a $80 \operatorname{anos}^{1}$. No Brasil, os registros epidemiológicos de prevalência e incidência de úlcera de perna são escassos e não há estimativas oficiais nos âmbitos nacional ou regionais.

Dentre as úlceras de perna, destaca-se a de etiologia venosa, também conhecida como úlcera de estase ou varicosa, que ocorre como resultado da hipertensão venosa e insuficiência da bomba do músculo gastrocnêmico9. É uma lesão de pele aberta, localizada na parte inferior da perna, entre o tornozelo e joelho, geralmente na região do maléolo medial, e que mostra pouco progresso para a cura no período de quatro a seis semanas do início de sua ocorrência ${ }^{5}$. Apresenta importância no contexto de saúde pública por acometer pessoas de diferentes faixas etárias e causar problemas socioeconômicos. Afeta o estilo de vida do paciente, pela necessidade de visitas clínicas ambulatoriais para trocas de curativos, 
dor crônica e odor desagradável pelo exsudato da lesão. O paciente precisa, com frequência, de cuidados de saúde, ocasionando afastamento do trabalho, aposentadoria precoce e sobrecarga econômica às instituições (pela queda da produtividade) $)^{10,11}$.

Para o tratamento de úlcera venosa, os profissionais utilizam diferentes terapias. Nos Estados Unidos da América, a bota de Unna é a mais aceita; no Reino Unido, a compressão elástica de multicamadas é amplamente utilizada; e na Europa continental, a bandagem de curto estiramento é prática padrão ${ }^{12}$.
A aplicação de pressão externa sobre o músculo da panturrilha ocasiona alterações capazes de influenciar positivamente o tratamento, tal como a elevação da pressão intersticial, que resulta no aumento do retorno venoso profundo, diminuindo o refluxo durante a deambulação, bem como no aumento do volume de ejeção durante a ativação dos músculos da panturrilha e da pressão tissular, favorecendo a reabsorção do edema e melhorando a drenagem linfática. Como consequência, as alterações desencadeadas pela ação da pressão facilitam a cura da úlcera varicosa $\mathrm{a}^{13,14}$.

\begin{tabular}{|c|c|}
\hline \multicolumn{2}{|r|}{ Avaliação e diagnóstico } \\
\hline \multirow{4}{*}{$A$} & $\Rightarrow$ Coletar o histórico do paciente; avaliar o paciente, a ferida, a pele periférica, a perna e o pé. \\
\hline & $\begin{array}{l}\Rightarrow \text { Realizar o índice de pressão tornozelo/braço (ITB) para avaliar a circulação arterial; encaminhar } \\
\text { para especialista para investigação e cuidados adicionais se ITB<0,8 ou >1,3. }\end{array}$ \\
\hline & $\Rightarrow$ Confirmar a presença de doença venosa (duplex scan). \\
\hline & $\begin{array}{l}\Rightarrow \text { Reavaliar se a redução de área da úlcera for menor que } 20 \text { a 30\% após } 4 \text { a } 6 \text { semanas de } \\
\text { tratamento de compressão ideal. }\end{array}$ \\
\hline \multicolumn{2}{|r|}{ Boa prática na gestão da pele e lesão } \\
\hline \multirow{5}{*}{$\mathrm{B}$} & $\begin{array}{l}\Rightarrow \text { Limpar, hidratar e proteger a pele periferida e a pele da perna: gerenciar a eczema e } \\
\text { hiperceratose, quando presentes. }\end{array}$ \\
\hline & $\Rightarrow$ Desbridar a lesão, se necessário e de acordo com o protocolo local. \\
\hline & $\begin{array}{l}\Rightarrow \text { Selecionar o tipo de cobertura e decidir a frequência de troca de curativo com base na } \\
\text { frequência da reaplicação de sistema de compressão e o nível de exsudato (a menos haja } \\
\text { suspeita ou a infecção esteja presente). }\end{array}$ \\
\hline & $\begin{array}{l}\Rightarrow \text { Utilizar coberturas antimicrobianas para infecção local ou para a prevenção da infecção em } \\
\text { lesões de alto risco (colonização crítica). }\end{array}$ \\
\hline & $\begin{array}{l}\Rightarrow \text { Assegurar o regime de compressão, a terapia da lesão e a adesão do paciente antes de } \\
\text { considerar terapias avançadas. }\end{array}$ \\
\hline \multicolumn{2}{|r|}{ Compressão } \\
\hline \multirow{7}{*}{ C } & $\begin{array}{l}\Rightarrow \text { Selecionar bandagem de compressão para otimizar o tratamento: inelásticos, elásticos. } \\
\text { O sistema de multicomponentes (combinação de elásticos e inelásticos) são preferíveis. }\end{array}$ \\
\hline & $\Rightarrow$ Atentar ao fato de que alguns pacientes podem necessitar de compressão modificada. \\
\hline & $\begin{array}{l}\Rightarrow \text { Considerar a utilização de meias de compressão para prevenção de recidiva ou para otimizar o } \\
\text { tratamento da úlcera quando o edema já estiver resolvido. }\end{array}$ \\
\hline & $\begin{array}{l}\Rightarrow \text { Encaminhar ao especialista para investigação e cuidados adicionais se considerar terapia de } \\
\text { compressão para pacientes com uma úlcera de etiologia mista com ITB<0,8 ou >1,3. }\end{array}$ \\
\hline & $\begin{array}{l}\Rightarrow \text { Não utilizar a terapia de compressão em pacientes com ITB<0,5: encaminhar ao cirurgião } \\
\text { vascular para possível revascularização. }\end{array}$ \\
\hline & $\Rightarrow$ Incentivar pacientes a serem ativos e a deambular. \\
\hline & $\Rightarrow$ Considerar compressão pneumática intermitente para pacientes totalmente imóveis. \\
\hline
\end{tabular}

Figura 2. Síntese das recomendações do Modelo ABC. 
A avaliação é uma etapa primordial que ampara a escolha do tratamento, incluindo a terapia de compressão, essencial para a cura da úlcera venosa e prevenção de sua recidiva. A terapia de compressão é uma terapia ativa

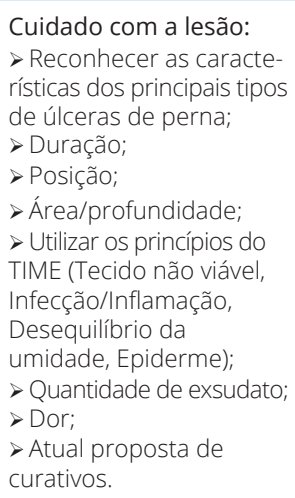

Pele ao redor da lesão:

> Maceração;

$>$ Escoriação;

$>$ Hiperceratose;

$>$ Sinais de doença

venosa (edema,

hiperpigmentação, eczema, dermatoesclersose).

$\Rightarrow$\begin{tabular}{|l|}
\hline Perna e pé: \\
> Evidência clínica de IVC \\
(coroa flebectásica no \\
tornozelo, varizes); \\
> Edema; \\
> Mobilidade do tornozelo; \\
> Pulsos nos pés; \\
> Doppler para avaliar a \\
circulação arterial (ex.: ITB); \\
> Duplex scan para avaliar \\
o sistema venoso \\
(quando disponível); \\
> Formato da perna; \\
> Sensação.
\end{tabular}

Sensação.
Úlcera venosa "simples"

$>$ ITB 0,8 - 1,3;

$>$ Área<100 cm²;

$>$ Tempo de existência

inferior a seis meses.

$$
\downarrow
$$

Gerenciamento no cuidado primário/nível de assistência primária:

Referenciar para o "Serviço Especializado de

Gerenciamento de úlcera Venosa" na ausência de profissionais competentes para utilização de terapia de compressão.
Pacientes com lesão de etiologia venosa ou

de outros fatores presentes, como diabetes, doença arterial e úlcera de etiologia mista (incluindo sempre a doença venosa).

\begin{tabular}{|c|c|}
\hline $\begin{array}{l}\text { Paciente: } \\
\text { > Histórico prévio de úlcera } \\
\text { de perna/doença venosa; } \\
\text { > Sintomas de IVC; } \\
\text { > Doenças associadas } \\
\text { (diabetes, doença cardíaca } \\
\text { ou cerebrovascular, insufi- } \\
\text { ciência arterial periférica); } \\
\text { > Alergias; } \\
\text { > Mobilidade; } \\
\text { > Destreza; } \\
\text { > Obesidade (IMC); }\end{array}$ & $\begin{array}{l}\text { Família e cuidadores: } \\
\text { > Entendimento } \\
\text { da condição; } \\
\text { > Vontade e habilidade } \\
\text { para oferecer suporte } \\
\text { (ex.: proximidade com } \\
\text { o paciente, meios de } \\
\text { transporte); } \\
\text { > Vontade e habilidade } \\
\text { para realizar a troca de } \\
\text { curativos e terapia de } \\
\text { compressão. }\end{array}$ \\
\hline
\end{tabular}

$>$ Impacto psicossocial da ferida;

$>$ Entendimento das

condições, expectativas e resultados esperados;

> Ocupação;

$>$ Autocuidado, cuidado e

suporte familiar;

> Experiência prévia e

adesão à terapia de

compressão;

> Transporte, capacidade

de ser atendido em clínica,

vontade e disponibilidade

de participar em

atendimento pelo telefone.

\section{(}

Úlcera venosa "complexa"

$>$ ITB 0,8 - 1,3;

$>$ Área< $100 \mathrm{~cm}^{2}$;

> Tempo de existência superior a seis meses;

> Insuficiência cardíaca controlada;

> Presença de infecção e/ou histórico de infecção recorrente;

$>$ Falta de adesão:

> Não redução de 20 a 30\% da área lesada em um prazo de 4 a 6 semanas, apesar da utilização de boas práticas.

\section{Referenciar para o serviço especializado que} gerencia a úlcera venosa: dependendo da provisão do serviço local, esse pode ser um serviço (clínica) especializado no gerenciamento de lesão, serviço comunitária com dermatologia, angiologia ou vascular. Investigações adicionais podem incluir duplex scan.
Sem evidência de que o paciente tenha IVC de forma que a etiologia contribua para a lesão; investigar e manejar de acordo com a etiologia apropriada.

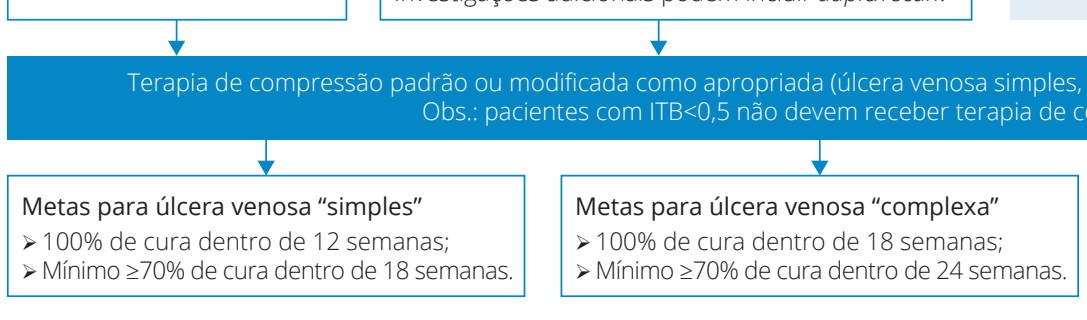

Etiologia mista na úlcera

$>$ ITB $0,8->1,3 *$;

$>$ Sintomas de doença arterial, tais como claudicação intermitente e dor ao repouso, mesmo se ITB estiver dentro da normalidade; $>$ Diabetes/neuropatia periférica:

$>$ Artrite reumatoide (úlcera vasculítica);

$>$ Insuficiência cardíaca descompesanda. $\downarrow$

Referenciar ao especialista apropriado para investigação e cuidado adicionais, como especialista vascular, angiologia, endocrinologia, reumatologia e cardiologia: Alguns pacientes podem ser manejados em colaboração de um serviço especializado que gerencie úlceras venosas. Investigações adicionais podem incluir duplex scan.

*Nota: se ITB $<0,5$, referenciar com urgência para possível revascularização. 
e geralmente subutilizada. No entanto, quando usada no paciente certo e de maneira correta para que seja mantida a adesão, ela é a chave para a cura de ulceração ativa. Avaliação e gestão devem ser executadas por um profissional de saúde que tenha recebido formação adequada. Se houver qualquer dúvida sobre a competência, o paciente deve ser encaminhado a um especialista. Otimizar a gestão de úlcera venosa utilizando o Modelo $\mathrm{ABC}$ contribuirá para reduzir o impacto financeiro que úlceras de perna causam nos sistemas de saúde de diversos países do mundo.

\section{CONCLUSÃO}

Espera-se que o Modelo proposto com abordagem simplificada ajude os profissionais a entenderem claramente por que, quando e como a terapia de compressão deve ser utilizada.

\section{REFERÊNCIAS}

1. Agale SV. [Internet] Chronic leg ulcers: epidemiology, aetiopathogenesis, and management [citado em 2015 abr 20]. Ulcers. 2013. Disponível em: http://www.hindawi.com/ journals/ulcers/2013/413604/

2. Puri N, Talwar A. Etiology and management of leg ulcers - an enigma. J Pakistan Assoc Dermatol. 2015;25(3):211-5.

3. Scottish Intercollegiate Guidelines Network. [Internet] Management of chronic venous leg ulcers:a national clinical guideline [citado em 2015 jul 04]. Edinburgh: Scottish Intercollegiate Guidelines Network (SIGN); 2010. Disponível em: http://www.sign.ac.uk/pdf/qrg120.pdf

4. Collins L, Seraj S. Diagnosis and treatment of venous ulcers. Am Fam Physician. 2010;81(8):989-96.

5. Harding K, Dowsett C, Fias L, Jelnes R, Mosti G, Öien R, et al. Simplifying venous leg ulcer management. Consensus recommendations. London: Wounds International; 2015.

6. Kahle B, Hermanns HJ, Gallenkemper G. Evidencebased treatment of chronic leg ulcers. Dtsch Arztebl Int. 2011;108(14):231-7.

7. Van Gent WB, Wilschut ED, Wittens C. Management of venous ulcer disease. BMJ. 2010;341(7782):1092-6.
8. Nelson EA, Bell-Syer SE. Compression for preventing recurrence of venous ulcers. Cochrane Database Syst Rev. 2012;(8):CD002303.

9. Johnson J, Paustian C. Guideline for management of wounds in patients with lower-extremity venous disease. Glenview: Wound, Ostomy, and Continence Nurses Society (WOCN); 2005.

10. Vishwanath V. Quality of life: venous leg ulcers. Indian Dermatol Online J. 2014;5(3):397-9.

11. Augustin M, Brocatti LK, Rustenbach SJ, Schäfer I, Herberger K. Cost-of-illness of leg ulcers in the community. Int Wound J. 2014;11(3):283-92

12. Cullum N, Nelson EA, Fletcher AW, Sheldon TA. Compression for venous leg ulcers. Cochrane Database Syst Rev. 2001;(2):CD000265.

13. Wound, Ostomy and Continence Nurses Society (WOCN). Guideline for management of wounds in patients with lowerextremity venous disease. Mount Laurel: WOCN; 2011.

14. White-Chu EF, Conner-Kerr TA. Overview of guidelines for the prevention and treatment of venous leg ulcers: a US perspective. J Multidiscip Healthc. 2014;7:111-7. 